\title{
An updated checklist of marine copepoda from Peninsular Malaysia with notes on their functional traits and distributional records
}

\author{
Balqis Balqiah Shafie ${ }^{1}$ and Azman Abdul Rahim ${ }^{* 1,2}$ \\ 1 Department of Earth Science and Environment, Faculty of Science and Technology, Universiti Kebangsaan \\ Malaysia, 43600 UKM Bangi, Selangor, Malaysia. \\ ${ }_{2}^{2}$ Marine Ecosystem Research Centre (EKOMAR), Faculty of Science and Technology, Universiti Kebangsaan \\ Malaysia, 43600 UKM Bangi, Selangor, Malaysia.
}

${ }^{*}$ Corresponding author: abarahim@ukm.edu.my

\begin{abstract}
An up-to-date checklist of marine zooplankton copepods from the waters of Peninsular Malaysia is presented. It contains 235 species of copepods consisting of 89 genera and 44 families. Each species is bibliographically referenced with synonymy, detailed information on their functional groups, and distributional data. The checklist is up to date as of 28 May 2020 and is based on taxonomic and ecological literature. Calanoida recorded the most diverse order, with 101 species, followed by Harpacticoida, with 70 species, and Cyclopoida, with 61 species. Canuelloida, Monstrilloida and Siphonostomatoida were all recorded with 1 species, respectively. As many as 192 species of copepods were recorded along the west coast (Malacca Strait) and 123 species al ong the east coast (South China Sea). Endemicity at the species level is $1 \%$ for the whole Peninsular Malaysian coast, with 2 genera restricted to the east coast (Kensakia parva Harris V.A. and Iwasaki, 1997 and Brachiella malayensis Ohtsuka, Piasecki, Ismail and Kamarudin, 2020) and 1 genus to the west coast (Labidocera jaafari Othman, 1986). Nine dominant species can be found along the coast of Peninsular Malaysia (Acartia erythraea Giesbrecht, 1889, Acartia pacifica Steuer, 1915, Bestiolina similis (Sewell, 1914), Euterpina acutifrons (Dana, 1848), Microsetella norvegica (Boeck, 1865), Paracalanus aculeatus Giesbrecht, 1888, Oithona nana Giesbrecht, 1893, Oithona simplex Farran, 1913 and Temora discaudata Giesbrecht, 1889).
\end{abstract}

Keywords: Copepoda, Zooplankton, Checklist, Distribution, Peninsular Malaysia

\section{Introduction}

Malaysia is situated in the Indo-Pacific region, which has a high zooplankton diversity (Yoshida et al., 2012), bounded on the west coast by the Malacca Straits and the east coast faces the South China Sea. Together with Indonesia and the Philippines, Malaysia is one of the three mega-diversity countries in the ASEAN region, blessed with several high biological productivity areas and extensive fishing grounds (Yoshida et al., 2012). The Southeast Asia region is home to more than 550 species of pelagic copepod, accounting for one-fourth of pelagic copepod species worldwide. Hence, this region is regarded as the centre of worldwide marine biodiversity (Nishida et al., 2011).

Copepoda is the most dominant group in the zooplankton community (Johan et al., 2013), and it is also acknowledged as the most abundant metazoan on earth (Alcaraz et al., 2003). Zooplankton also acts as an ecological indicator (Metillo et al., 2019), responsive to changes in water parameters such as salinity, $\mathrm{pH}$ and temperature (Shuaib et al., 2019). Changes in zooplankton may disrupt and alter the food chain, having a detrimental effect on not only fisheries yield but also on their stability and sustainability, considering that this group (zooplankton) occupies the secondary trophic level that connects primary producers to higher trophic levels (Alcaraz et al., 2003; Shuaib et al., 2018).

According to Yoshida et al. (2012), Sewell (1933) was the first researcher to study copepods in Malaysian waters in the Straits of Malacca, collecting calanoid copepod from Penang and Sungai Kurau Estuary in Perak. Wickstead (1961) then completed an extensive plankton collection in the Malacca Straits-Singapore Straits-South China Sea areas. On the other hand, Othman (1988) was the earliest to study copepods along the east coast of Peninsular Malaysia, concentrating primarily on the Exclusive Economic Zone (EEZ) in the South China Sea and identified 74 species of copepods. Hence, the objective of this paper was to compile and update the checklist of this noteworthy zooplankton group found in the waters of Peninsular Malaysia.

\section{Materials and Methods}

The checklist is based on taxonomic and ecological literature reviewed until 28 May 2020 for species recorded in the coastal waters of Peninsular Malaysia (Figure 1). The data were obtained via an intensive literature review (130 published papers were extracted 
roughly) concentrating on recorded copepod, published from Peninsular Malaysia at the species level. Some biodiversity knowledge can be accessed through databases such as World Register of Marine Species WoRMS (http://www.marinespecies.org), Biodiversity of Marine Planktonic Copepod (https://copepodes.obsbanyuls.fr/en/index.php), and the University of Tasmania, Australia - Zooplankton (https://www.imas.utas.edu.au/zooplankton/download able-species-fact-sheets). The family and species in the checklist were arranged following Rezai et al. (2004). Within a family, the species and genera were alphabetically ordered. References and distribution records of the checked species were detailed, including their spawning strategies, feeding method and biogeographical distribution for those species with expanded range of distribution, beyond Malaysian waters.

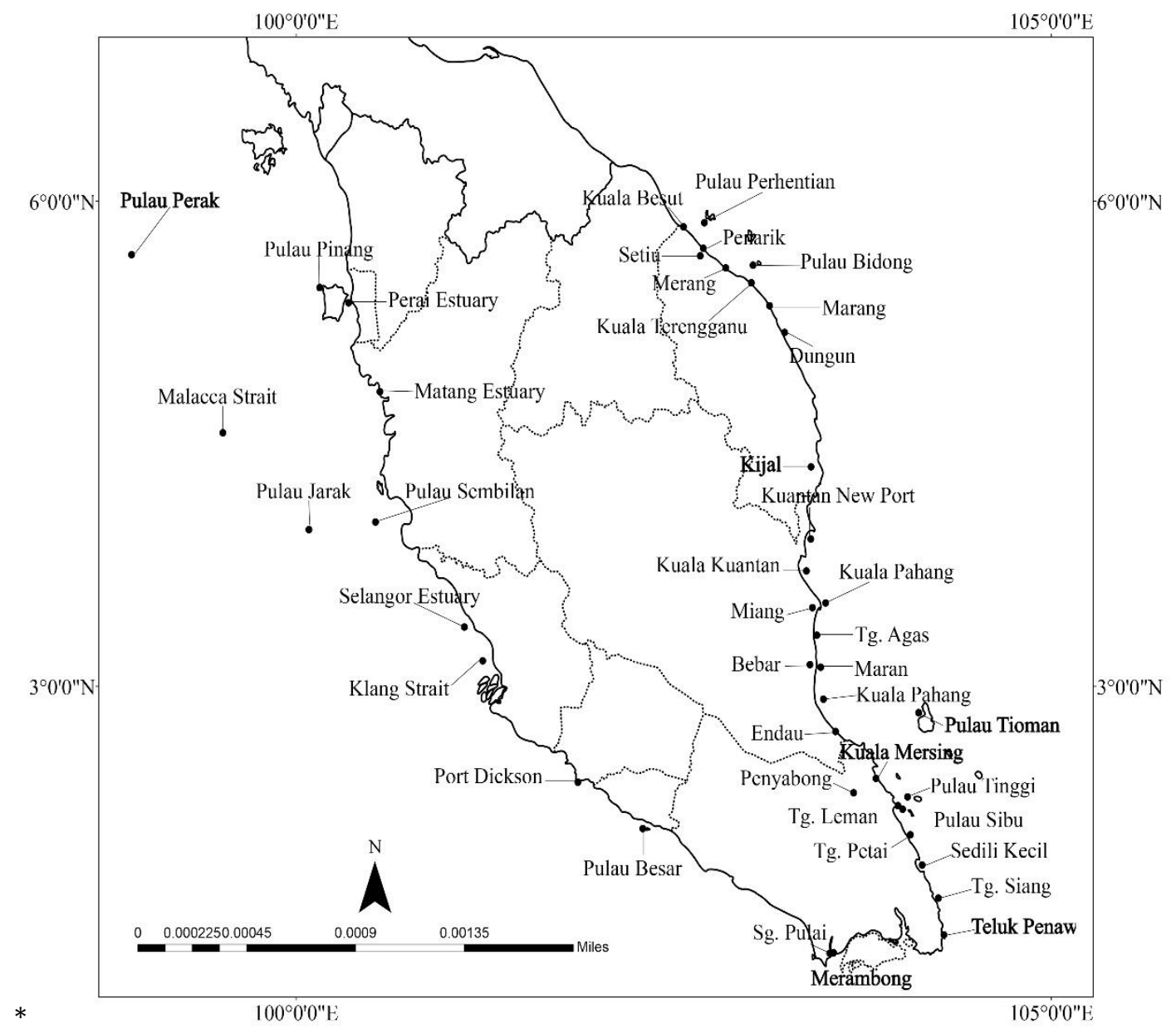

Figure 1. A map of Peninsular Malaysia with localities considered in the present study.

Table 1 contains the detailed information: The hierarchical taxonomic position of the family (in alphabetical order), indicating the following data: a) list of species synonyms (in superscript); b) distributional characteristic of the taxa; c) spawning strategies which are broadcaster (individuals continuously releasing eggs or as clutches on a daily basis (Hopcroft and Roff, 1996) and sac-spawner (individuals that carry their eggs in sac until hatching (Kiørboe and Sabatini, 1994); d) trophic group (i.e. Carnivorous, Herbivorous, Omnivorous, Detritivorous, Omnivorous-Carnivorous, HerbivorousOmnivorous, and
Omnivorous-Detritivorous (Benedetti et al., 2018, Campos et al., 2017, Nakajima et al., 2014); e) feeding method, including ambush, cruise, filter-feeders and mixed (species which can switch their feeding strategies based on food availability) (Kiørboe, 2011), scrapers (Heinle, 2013) and predator (Uye, 1994; f) biogeographic distribution of copepod fauna based of their region suitability (i.e. Indian Ocean, Atlantic, Pacific and others) (Carola, 1994). Nomenclature follows recent taxonomic changes tracked using the WoRMS (World Register of Marine Species, hosted by the Belgian Institute of Marine Science (VLIZ), accessed in November 2020). 
Table 1. An updated checklist (November 2020) of the order of Copepoda Milne Edwards, 1840 recorded from Peninsular Malaysia.

\begin{tabular}{|c|c|c|c|c|}
\hline FAMILY/SPECIES & $\begin{array}{l}\text { SPAWNING } \\
\text { STRATEGY }\end{array}$ & $\begin{array}{l}\text { TROPHIC } \\
\text { GROUP }\end{array}$ & $\begin{array}{l}\text { FEEDING } \\
\text { METHOD }\end{array}$ & $\begin{array}{l}\text { GENERAL } \\
\text { BIOGEOGRAPHY }\end{array}$ \\
\hline \multicolumn{5}{|l|}{ ACARTIIDAE } \\
\hline $\begin{array}{l}\text { Acartia amboinensis } \\
(\text { Carl, 1907) })^{1,27,31,36}\end{array}$ & Broadcaster & $\begin{array}{l}\text { Herbivorous- } \\
\text { omnivorous }\end{array}$ & NA & SEA, SWP, ID \\
\hline $\begin{array}{l}\text { Acartia bispinosa } \\
(\text { Carl, 1907) }\end{array}$ & Broadcaster & Omnivorous & NA & ID, NWP, SWP \\
\hline $\begin{array}{l}\text { Acartia danae } \\
\text { Giesbrecht, } 1889^{31}\end{array}$ & Broadcaster & Omnivorous & Mixed & Atl, ID, Pacific, A \\
\hline $\begin{array}{l}\text { Acartia erythraea } \\
\text { Giesbrecht,18891,2,3,4,5,20,22,23,26,27,28,35,36 }\end{array}$ & Broadcaster & Omnivorous & NA & Nat, ID, NWP, SWP \\
\hline $\begin{array}{l}\text { Acartia pacifica } \\
\text { Steuer, } 19151,20,23,26,27,28,31,34,35,36\end{array}$ & Broadcaster & Omnivorous & NA & $\begin{array}{l}\text { Pacific, ID, Tropical } \\
\text { and } \\
\text { Sub-tropical }\end{array}$ \\
\hline $\begin{array}{l}\text { Acartia spinicauda } \\
\text { Giesbrecht, } 18891,2,3,4,5,9,11,19,27,34,35,36\end{array}$ & Broadcaster & Omnivorous & NA & SWP, ID \\
\hline \multicolumn{5}{|l|}{ AMEIRIDAE } \\
\hline $\begin{array}{l}\text { Nitocra spinipes armata } \\
\text { Lang, } 1965^{12,13}\end{array}$ & Sac-spawner & $\begin{array}{l}\text { Detrivorous- } \\
\text { herbivorous }\end{array}$ & NA & NEA, ID, NWP \\
\hline $\begin{array}{l}\text { Nitocra typica } \\
\text { Boeck, } 1865^{12,13} \\
\end{array}$ & Sac-spawner & Herbivorous & NA & NA, ID \\
\hline \multicolumn{5}{|l|}{ ARITELLIDAE } \\
\hline $\begin{array}{l}\text { Metacalanus aurivilli } \\
\text { Cleve, } 1901^{2,3,4,5,20}\end{array}$ & Broadcaster & Carnivorous & NA & ID, NWP \\
\hline \multicolumn{5}{|l|}{ CANDACIIDAE } \\
\hline $\begin{array}{l}\text { Candacia bradyi } \\
\text { Scott A., 190220,23,27 }\end{array}$ & Broadcaster & Carnivorous & NA & ID, SWP, NWP, SEA \\
\hline $\begin{array}{l}\text { Candacia catula } \\
\text { Giesbrecht, } 1889^{1,23}\end{array}$ & Broadcaster & Carnivorous & $\begin{array}{l}\text { Piercing } \\
\text { and } \\
\text { sucking }\end{array}$ & $\begin{array}{l}\text { ID, SEA, NEA, } \\
\text { NWP, SWP }\end{array}$ \\
\hline $\begin{array}{l}\text { Candacia curta } \\
(\text { Dana, 1849) })^{27}\end{array}$ & Broadcaster & Carnivorous & NA & Atl, ID, Pacific \\
\hline $\begin{array}{l}\text { Candacia discaudata } \\
\text { Scott A., 19091,3,5,27 }\end{array}$ & Broadcaster & Carnivorous & NA & ID, NP \\
\hline $\begin{array}{l}\text { Candacia ethiopica } \\
\text { (Dana, 1849) }\end{array}$ & Broadcaster & Carnivorous & Ambush & ID, Pacific, NA \\
\hline $\begin{array}{l}\text { Candacia pachydactyla } \\
\text { (Dana, 1849) }\end{array}$ & Broadcaster & Carnivorous & $\begin{array}{l}\text { Piercing } \\
\text { and } \\
\text { sucking }\end{array}$ & $\begin{array}{l}\text { Atl, ID, NWP, } \\
\text { Sub-Antarctic }\end{array}$ \\
\hline $\begin{array}{l}\text { Canthocalanuspauper } \\
\text { (Giesbrecht, 1888) }^{1,3,4,5,9,20,21,23,26,27,28}\end{array}$ & NA & Herbivorous & $\begin{array}{l}\text { Filter- } \\
\text { feeders }\end{array}$ & ID, Pacific, SWA \\
\hline 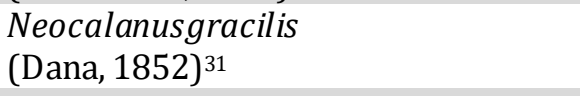 & Broadcaster & Herbivorous & $\begin{array}{l}\text { Filter- } \\
\text { feeders }\end{array}$ & ID, Pacific, NA \\
\hline $\begin{array}{l}\text { Nannocalanus minor } \\
\text { (Claus, } 1863)^{23,26,27}\end{array}$ & Broadcaster & $\begin{array}{l}\text { Herbivorous- } \\
\text { omnivorous }\end{array}$ & $\begin{array}{l}\text { Filter- } \\
\text { feeders }\end{array}$ & AA, ID, NA, Pacific \\
\hline $\begin{array}{l}\text { Undinula vulgaris } \\
\text { (Dana, 1849) }\end{array}$ & Broadcaster & $\begin{array}{l}\text { Herbivorous- } \\
\text { omnivorous }\end{array}$ & $\begin{array}{l}\text { Filter- } \\
\text { feeders }\end{array}$ & ID, SEA, NA, Pacific \\
\hline \multicolumn{5}{|l|}{ CANUELLIDAE } \\
\hline $\begin{array}{l}\text { Brianolastebleri } \\
\text { (Monad, 1926) }^{10,13}\end{array}$ & Sac-spawner & NA & NA & $\begin{array}{l}\text { Mediterranean Sea, } \\
\text { ID }\end{array}$ \\
\hline \multicolumn{5}{|l|}{ CENTROPAGIDAE } \\
\hline $\begin{array}{l}\text { Centropages dorsispinatus Thompson } \\
\text { I.C. and ScottA., 19031,2,3,4,5,9,27 }\end{array}$ & Broadcaster & Omnivorous & NA & ID, NWP \\
\hline
\end{tabular}




\begin{tabular}{|c|c|c|c|c|}
\hline $\begin{array}{l}\text { Centropages furcatus } \\
\text { (Dana, 1849) }^{1,3,4,5,11,20,23,26,27,29}\end{array}$ & Broadcaster & Omnivorous & Mixed & A, Atl, ID, Pacific \\
\hline $\begin{array}{l}\text { Centropages orsinii } \\
\text { Giesbrecht, } 18891,20,23,26,27\end{array}$ & Broadcaster & Omnivorous & NA & ID, SWP, NWP \\
\hline $\begin{array}{l}\text { Centropages sinensis } \\
\text { Chen and Zhang, } 1965^{26}\end{array}$ & Broadcaster & Omnivorous & NA & NWP \\
\hline $\begin{array}{l}\text { Centropages tenuiremis } \\
\text { Thompson I.C. and ScottA., 19035,20,26,27 }\end{array}$ & Broadcaster & Omnivorous & NA & ID, NWP \\
\hline \multicolumn{5}{|l|}{ CLAUSOCALANIDAE } \\
\hline 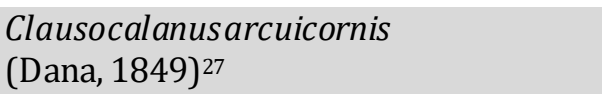 & Sac-spawner & $\begin{array}{l}\text { Detrivorous- } \\
\text { herbivorous }\end{array}$ & $\begin{array}{l}\text { Filter- } \\
\text { feeders }\end{array}$ & ID, SEP, NP, NA \\
\hline $\begin{array}{l}\text { Clausocalanusfarrani } \\
\text { Sewell, } 1929^{26}\end{array}$ & Sac-spawner & Herbivorous & NA & ID, NEA, NP, SEP \\
\hline $\begin{array}{l}\text { Clausocalanusfurcatus } \\
\text { (Brady, 1883) }{ }^{26,27}\end{array}$ & Sac-spawner & Herbivorous & $\begin{array}{l}\text { Filter- } \\
\text { feeders }\end{array}$ & Atl, Pacific, ID \\
\hline $\begin{array}{l}\text { Clausocalanusjobei } \\
\text { Frost and Fleminger, } 1968^{27}\end{array}$ & Sac-spawner & Herbivorous & $\begin{array}{l}\text { Filter- } \\
\text { feeders }\end{array}$ & NWP, NP, SEP, ID \\
\hline $\begin{array}{l}\text { Clausocalanusminor } \\
\text { Sewell, } 192926\end{array}$ & Sac-spawner & Herbivorous & NA & SWP, NWP, ID \\
\hline $\begin{array}{l}\text { Clausocalanuspergens } \\
\text { Farran, } 1926^{27}\end{array}$ & Sac-spawner & Herbivorous & NA & NA, Pacific, ID \\
\hline \multicolumn{5}{|l|}{ CLETODIDAE } \\
\hline $\begin{array}{l}\text { Enhydrosoma longifurcatum } \\
\text { Sars G.0., } 190932\end{array}$ & NA & NA & NA & ID, WCP, Atl \\
\hline \multicolumn{5}{|l|}{ CORYCAEIDAE } \\
\hline $\begin{array}{l}\text { Corycaeus affinis } \\
\text { McMurrich, } 1916^{1,27}\end{array}$ & Sac-spawner & Carnivorous & NA & ID, NP, SWA \\
\hline $\begin{array}{l}\text { Corycaeus agilis } \\
\text { Dana, } 1849^{1,27,31}\end{array}$ & Sac-spawner & Carnivorous & NA & NA, ID, Pacific \\
\hline $\begin{array}{l}\text { Corycaeus andrewsi } \\
\text { Farran, } 1911^{1,2,3,4,9,20,27}\end{array}$ & Sac-spawner & Carnivorous & Predator & ID, NWP \\
\hline $\begin{array}{l}\text { Corycaeus asiaticus } \\
\text { Dahl F., } 18941,20,27,31\end{array}$ & Sac-spawner & Carnivorous & Predator & SEA, ID, NWP, NEA \\
\hline $\begin{array}{l}\text { Corycaeus catus } \\
\text { Dahl F., 18941,20,27 }\end{array}$ & Sac-spawner & Carnivorous & NA & NA, ID, NP, SEP \\
\hline $\begin{array}{l}\text { Corycaeus crassiusculus } \\
\text { Dana, } 1849^{20,26,31}\end{array}$ & Sac-spawner & Carnivorous & Predator & NA, ID, NWP, SEP \\
\hline $\begin{array}{l}\text { Corycaeus dahli } \\
\text { Tanaka, } 18571,3,4,9,20,27\end{array}$ & Sac-spawner & Carnivorous & Predator & SEA, ID, NWP \\
\hline $\begin{array}{l}\text { Corycaeus dubius } \\
\text { Farran, } 1911^{27}\end{array}$ & Sac-spawner & Carnivorous & NA & SEP \\
\hline $\begin{array}{l}\text { Corycaeus erythraeus } \\
\text { Cleve, } 1904^{1,3,4,20,27}\end{array}$ & Sac-spawner & Carnivorous & NA & NEA, ID, NWP, SEP \\
\hline $\begin{array}{l}\text { Corycaeus latus } \\
\text { Dana, } 1849^{20}\end{array}$ & Sac-spawner & Carnivorous & NA & SEA, NA, ID, Pacific \\
\hline $\begin{array}{l}\text { Corycaeus lautus } \\
\text { Dana, } 1849^{1,27}\end{array}$ & Sac-spawner & Carnivorous & NA & NA, ID, NP, SEP \\
\hline $\begin{array}{l}\text { Corycaeus limbatus } \\
\text { Brady, } 1883^{1,27}\end{array}$ & Sac-spawner & Carnivorous & Ambush & NA, ID, NP, SEP \\
\hline $\begin{array}{l}\text { Corycaeus longistylis } \\
\text { (Dana, 1849) } 26,31\end{array}$ & Sac-spawner & Carnivorous & NA & NA, ID, Pacific \\
\hline $\begin{array}{l}\text { Corycaeus lubbocki } \\
\text { Giesbrecht, } 1891^{20}\end{array}$ & Sac-spawner & Carnivorous & Predator & NA, ID, NP \\
\hline $\begin{array}{l}\text { Corycaeus pacificus } \\
\text { Dahl F., 189426,27,31 }\end{array}$ & Sac-spawner & Carnivorous & NA & SEA, NA, ID, NWP \\
\hline $\begin{array}{l}\text { Corycaeus pumilus } \\
\text { Dahl M., } 1912^{1}\end{array}$ & Sac-spawner & Carnivorous & NA & SEA, NEA, ID, NWP \\
\hline
\end{tabular}




\begin{tabular}{|c|c|c|c|c|}
\hline $\begin{array}{l}\text { Corycaeus speciosus } \\
\text { Dana, } 18491,3,5,20,26,27,31\end{array}$ & Sac-spawner & Carnivorous & NA & SWA, NA, ID, Pacific \\
\hline $\begin{array}{l}\text { Corycaeus robustus } \\
\text { Giesbrecht, } 1892^{31}\end{array}$ & Sac-spawner & Carnivorous & NA & NA, ID, SEP, NWP \\
\hline $\begin{array}{l}\text { Corycaeus subtilis } \\
\text { Dahl M., } 1912^{9,27}\end{array}$ & Sac-spawner & Carnivorous & Predator & NEA, ID, NWP \\
\hline $\begin{array}{l}\text { Ditrichocorycaeus affinis } \\
(\text { McMurrich, 1916) }\end{array}$ & Sac-spawner & Carnivorous & NA & ID, NP \\
\hline $\begin{array}{l}\text { Ditrichocorycaeus andrewsi } \\
\text { (Farran, 1911) }\end{array}$ & Sac-spawner & Carnivorous & Predator & ID, NWP, SWP \\
\hline $\begin{array}{l}\text { Ditrichocorycaeus asiaticus } \\
\text { (Dahl F., 1894) }\end{array}$ & Sac-spawner & Carnivorous & Predator & ID, NWP, SWP, NWA \\
\hline $\begin{array}{l}\text { Ditrichocorycaeusdahli } \\
\text { (Tanaka, 1957) }\end{array}$ & Sac-spawner & Carnivorous & Predator & ID, NWP, SWP \\
\hline $\begin{array}{l}\text { Ditrichocorycaeus erythraeus } \\
(\text { Cleve, } 1904)^{5}\end{array}$ & Sac-spawner & Carnivorous & NA & $\begin{array}{l}\text { ID, NWP, SWP, } \\
\text { SEP, SWA }\end{array}$ \\
\hline $\begin{array}{l}\text { Farranula concinna } \\
(\text { Dana, 1849) }\end{array}$ & Sac-spawner & Carnivorous & NA & NWA, SEA, ID, NWP \\
\hline $\begin{array}{l}\text { Farranula gibbula } \\
\text { (Giesbrecht, 1891) }^{20,21,27}\end{array}$ & Sac-spawner & Carnivorous & NA & NP, SEP, ID \\
\hline $\begin{array}{l}\text { Farranula rostratus } \\
(\text { Claus, } 1863)^{27}\end{array}$ & Sac-spawner & Carnivorous & NA & NA, ID, NP, SEP \\
\hline $\begin{array}{l}\text { Onychocorycaeuscatus } \\
\text { (Dahl F., 1894) }\end{array}$ & Sac-spawner & Carnivorous & NA & NWA, ID, NWP, SWP \\
\hline $\begin{array}{l}\text { Onychocorycaeus pumilus } \\
\text { (Dahl M., 1912) }^{5}\end{array}$ & Sac-spawner & Carnivorous & NA & NEA, ID, NWP, SWP \\
\hline \multicolumn{5}{|l|}{ CYCLOPIDAE } \\
\hline $\begin{array}{l}\text { Apocyclops ramkhamhaengi } \\
\text { Chullasorn, Kangtia, } \\
\text { Pinkaew and Ferrari, } 2008^{33}\end{array}$ & Broadcaster & Omnivorous & NA & WCP \\
\hline \multicolumn{5}{|l|}{ DACTYLOPUSIIDAE } \\
\hline $\begin{array}{l}\text { Dactylopusia crassicornis } \\
\text { Brady, } 1910^{13}\end{array}$ & Sac-spawner & NA & NA & $\begin{array}{l}\text { The Southern Ocean, } \\
\text { WCP }\end{array}$ \\
\hline $\begin{array}{l}\text { Dactylopusia crassipes } \\
\text { (Lang, 1965) }^{10,13}\end{array}$ & Sac-spawner & Herbivorous & NA & ID \\
\hline $\begin{array}{l}\text { Diarthrodes tetrastachyus } \\
\text { Yeatman, } 1976^{12,13}\end{array}$ & NA & NA & NA & NA, NWP \\
\hline $\begin{array}{l}\text { Paradactylopodia oculata } \\
\text { (Gurney, 1927) }\end{array}$ & NA & NA & NA & Cosmopolite \\
\hline \multicolumn{5}{|l|}{ DARCYTHOMPSONIIDAE } \\
\hline $\begin{array}{l}\text { Darcythompsonia fairliensis } \\
\text { (Scott T., 1899) } 32\end{array}$ & NA & NA & NA & NA, NWP \\
\hline $\begin{array}{l}\text { Darcythompsonia inopinata } \\
\text { Smirnov, } 1934^{32}\end{array}$ & NA & NA & NA & ID, WCP, NA \\
\hline \multicolumn{5}{|l|}{ ECTINOSOMATIDAE } \\
\hline $\begin{array}{l}\text { Ectinosoma melaniceps } \\
\text { Boeck, } 1865^{12,13}\end{array}$ & Sac-spawner & $\begin{array}{l}\text { Detritivorou- } \\
\text { herbivorous }\end{array}$ & NA & NEA, ID \\
\hline $\begin{array}{l}\text { Halectinosoma brunneum } \\
\text { (Brady, 1905) }^{10,13}\end{array}$ & Sac-spawner & $\begin{array}{l}\text { Detritivorou- } \\
\text { herbivorous }\end{array}$ & NA & NA, NWP \\
\hline $\begin{array}{l}\text { Microsetella norvegica } \\
\text { (Boeck, 1865) }^{1,2,3,4,5,9,11,20,21,23,27,31}\end{array}$ & Sac-spawner & $\begin{array}{l}\text { Detritivorou- } \\
\text { herbivorous }\end{array}$ & Predator & AA, A, ID, NWP \\
\hline $\begin{array}{l}\text { Microsetella rosea } \\
\text { (Dana, 1847) }{ }^{11,20,27}\end{array}$ & Sac-spawner & $\begin{array}{l}\text { Detrivorous- } \\
\text { omnivorous }\end{array}$ & Predator & AA, NA, ID, Pacific \\
\hline \multicolumn{5}{|l|}{ EUCALANIDAE } \\
\hline $\begin{array}{l}\text { Eucalanus attenuatus } \\
\text { (Dana, 1849) } \\
1,27\end{array}$ & Broadcaster & Herbivorous & NA & ID, Pacific \\
\hline
\end{tabular}




\begin{tabular}{|c|c|c|c|c|}
\hline $\begin{array}{l}\text { Eucalanus crassus } \\
\text { Giesbrecht, } 1888^{27,28}\end{array}$ & Broadcaster & Herbivorous & NA & NA, ID, NWP, SEP \\
\hline $\begin{array}{l}\text { Eucalanus elongatus } \\
\text { (Dana, 1848) }\end{array}$ & Broadcaster & Herbivorous & $\begin{array}{l}\text { Filter- } \\
\text { feeders }\end{array}$ & ID, NWP, SEP \\
\hline $\begin{array}{l}\text { Eucalanus pileatus } \\
\text { Giesbrecht, } 1888^{27}\end{array}$ & Broadcaster & Herbivorous & NA & SWA, NA, ID, NP \\
\hline $\begin{array}{l}\text { Eucalanus subcrassus } \\
\text { Giesbrecht, } 1888^{1,3,4,9,27,28}\end{array}$ & Broadcaster & Herbivorous & NA & NA, ID, NP, SEP \\
\hline $\begin{array}{l}\text { Eucalanus subtenuis } \\
\text { Giesbrecht, } 1888^{1,9,27}\end{array}$ & Broadcaster & Herbivorous & NA & NWA, ID, NP, SEP \\
\hline \multicolumn{5}{|l|}{ EUCHAETIDAE } \\
\hline $\begin{array}{l}\text { Euchaeta concinna } \\
\text { Dana, } 18491,5,27\end{array}$ & Broadcaster & Carnivorous & NA & NEA, ID, NWP, SEP \\
\hline $\begin{array}{l}\text { Euchaeta marinella } \\
\text { Bradford, } 1974^{27,29}\end{array}$ & Broadcaster & Carnivorous & NA & ID, NWP, SEP, SWP \\
\hline $\begin{array}{l}\text { Euchaeta wolfendeni } \\
\text { Scott A., } 1909^{1}\end{array}$ & Broadcaster & Carnivorous & NA & SWP, ID, NWP, SEP \\
\hline $\begin{array}{l}\text { Paraeuchaeta concinna } \\
\text { (Dana, 1849) }{ }^{23}\end{array}$ & NA & NA & NA & Atl, ID, NWP \\
\hline \multicolumn{5}{|l|}{ HARPACTICIDAE } \\
\hline $\begin{array}{l}\text { Harpacticus clausi } \\
\text { Scott A., } 190913\end{array}$ & NA & NA & NA & ID, WCP \\
\hline $\begin{array}{l}\text { Harpacticus spinulosus } \\
\text { Lang, } 1965^{12,13}\end{array}$ & NA & Herbivorous & NA & NWP \\
\hline $\begin{array}{l}\text { Harpacticus uniremis } \\
\text { Krøyer in Gaimard, 1842-1845? }{ }^{12,13}\end{array}$ & NA & Herbivorous & NA & Atl \\
\hline \multicolumn{5}{|l|}{ IDYANTHIDAE } \\
\hline $\begin{array}{l}\text { Idyanthe pusilla } \\
\text { (Sars G.O., 1905) }^{16} \\
\end{array}$ & NA & NA & NA & NA, NWP \\
\hline \multicolumn{5}{|l|}{ LAOPHONTIDAE } \\
\hline $\begin{array}{l}\text { Heterolaophonte longifurcata } \\
\text { Lang, } 1965^{12,13}\end{array}$ & NA & Herbivorous & NA & SWP, NWP \\
\hline $\begin{array}{l}\text { Laophonte cornuta } \\
\text { Philippi, } 1840^{30}\end{array}$ & NA & Herbivorous & NA & NEA, NWP \\
\hline $\begin{array}{l}\text { Laophonte expansa } \\
\text { Fiera, } 19866^{17}\end{array}$ & NA & Herbivorous & NA & ID, NWP \\
\hline $\begin{array}{l}\text { Paralaophonte brevirostris } \\
\text { (Claus, 1863) } 12,13\end{array}$ & NA & Herbivorous & NA & Atl, NWP \\
\hline $\begin{array}{l}\text { Paralaophonte octavia } \\
\text { (Monard, } 1935)^{7,10,13}\end{array}$ & NA & Herbivorous & NA & Atl, NWP \\
\hline $\begin{array}{l}\text { Quinquelaophonte quinquespinosa } \\
\text { (Sewell, 1924) }{ }^{17}\end{array}$ & NA & NA & NA & WCP \\
\hline \multicolumn{5}{|l|}{ LERNAEOPODIDAE } \\
\hline $\begin{array}{l}\text { Brachiellamalayensis } \\
\text { Ohtsuka, Piasecki, Ismail and } \\
\text { Kamarudin, } 2020^{24}\end{array}$ & NA & NA & NA & Endemic to Malaysia \\
\hline \multicolumn{5}{|l|}{ LONGIPEDIIDAE } \\
\hline $\begin{array}{l}\text { Longipedia weberi } \\
\text { Scott A., 190912,13 }\end{array}$ & NA & NA & NA & Atl, ID, NWP, WCP \\
\hline \multicolumn{5}{|l|}{ LOURINIIDAE } \\
\hline $\begin{array}{l}\text { Lourinia armata } \\
\text { (Claus, 1866) }{ }^{12,13}\end{array}$ & NA & NA & NA & Atl, NWP \\
\hline \multicolumn{5}{|l|}{ LUCICUTIIDAE } \\
\hline $\begin{array}{l}\text { Lucicutiaflavicornis } \\
\text { (Claus, 1963) } 5,27,29\end{array}$ & Broadcaster & $\begin{array}{l}\text { Herbivorous- } \\
\text { omnivorous }\end{array}$ & $\begin{array}{l}\text { Filter- } \\
\text { feeders }\end{array}$ & NA, ID, Pacific, AA \\
\hline $\begin{array}{l}\text { Lucicutiagaussae } \\
\text { Grice, } 1963^{1,5,27}\end{array}$ & Broadcaster & $\begin{array}{l}\text { Herbivorous- } \\
\text { omnivorous }\end{array}$ & NA & NA, ID, NWP, SEP \\
\hline
\end{tabular}




\section{MACROCHIRONIDAE}

\begin{tabular}{|c|c|c|c|c|}
\hline $\begin{array}{l}\text { Paramacrochiron amboinense } \\
\text { Mulyadi, } 2005^{3,4}\end{array}$ & NA & NA & NA & $\begin{array}{l}\text { The South China Sea, } \\
\text { Java Sea }\end{array}$ \\
\hline \multicolumn{5}{|l|}{ METIDAE } \\
\hline $\begin{array}{l}\text { Metis jousseaumei } \\
\text { (Richard, 1892) }^{12,13}\end{array}$ & NA & NA & NA & ID, NA \\
\hline \multicolumn{5}{|l|}{ MIRACIIDAE } \\
\hline $\begin{array}{l}\text { Amphiascoidessubdebilis } \\
\text { (Willey, 1935) }\end{array}$ & NA & NA & NA & NA, NWP \\
\hline $\begin{array}{l}\text { Amphiascopsis coralicola } \\
\text { (Sewell, 1940) }\end{array}$ & NA & NA & NA & NA, NWP \\
\hline $\begin{array}{l}\text { Amphiascopsis thalestroides } \\
\text { (Sars G.O., 1911)12,13 }\end{array}$ & NA & NA & NA & ID, NA \\
\hline 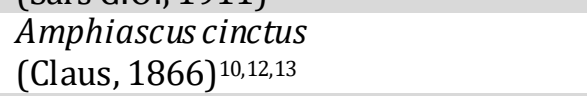 & NA & Herbivorous & NA & NWP, NA \\
\hline $\begin{array}{l}\text { Amphiascus rebus } \\
\text { Sewell, } 1940^{12,13}\end{array}$ & NA & Herbivorous & NA & ID, NA, NWP \\
\hline $\begin{array}{l}\text { Amphiascus robinsonii } \\
\text { (Scott A., 1902) }^{12,13}\end{array}$ & NA & Herbivorous & NA & NA, WCP \\
\hline $\begin{array}{l}\text { Delavalia clavus } \\
\text { (Wells and Rao, 1987) }\end{array}$ & NA & NA & NA & ID \\
\hline $\begin{array}{l}\text { Distioculus minor } \\
\text { (Scott T., 1894) }\end{array}$ & Sac-spawner & Carnivorous & NA & NA, ID \\
\hline $\begin{array}{l}\text { Macrosetella gracilis } \\
\text { (Dana, 1864) } 1,3,4,20,21,23,26,27,31\end{array}$ & Sac-spawner & $\begin{array}{l}\text { Herbivorous- } \\
\text { omnivorous }\end{array}$ & $\begin{array}{l}\text { Filter- } \\
\text { feeders }\end{array}$ & Atl, ID, NP \\
\hline $\begin{array}{l}\text { Metamphiascopsis hirsutus } \\
\text { (Thompson I.C. and Scott A., 1903) }\end{array}$ & NA & NA & NA & WCP \\
\hline $\begin{array}{l}\text { Metamphiascopsis hirsutus bermudae } \\
\text { (Willey, 1930) }{ }^{13}\end{array}$ & NA & NA & NA & WCP \\
\hline $\begin{array}{l}\text { Miracia efferata } \\
\text { Dana, } 1849^{26}\end{array}$ & Sac-spawner & $\begin{array}{l}\text { Herbivorous- } \\
\text { omnivorous }\end{array}$ & NA & Atl, ID, NWP \\
\hline $\begin{array}{l}\text { Paramphiascella calcarifer } \\
(\text { Sewell, 1940 })^{32}\end{array}$ & NA & NA & NA & Atl, WCP \\
\hline $\begin{array}{l}\text { Paramphiascellarobinsonii } \\
\text { (Scott A., 1902) }\end{array}$ & NA & NA & NA & ID, WCP \\
\hline $\begin{array}{l}\text { Rhyncholagenalevantina } \\
\text { Por, } 1964^{10,13}\end{array}$ & NA & NA & NA & NA, NWP \\
\hline $\begin{array}{l}\text { Robertgurneya diversa } \\
\text { (Lang, 1965) })^{12,13}\end{array}$ & NA & NA & NA & NWA, NWP \\
\hline $\begin{array}{l}\text { Robertgurneya oligochaeta } \\
\text { (Noodt, 1955) }\end{array}$ & NA & NA & NA & NA, NWP \\
\hline $\begin{array}{l}\text { Robertgurneya smithi } \\
\text { Hamond, } 1973^{29}\end{array}$ & NA & NA & NA & NEA, WCP \\
\hline $\begin{array}{l}\text { Robertsonia knoxi } \\
\text { (Thompson I.C. and Scott A., 1903) } 14\end{array}$ & NA & NA & NA & WCP \\
\hline $\begin{array}{l}\text { Schizopera knabeni } \\
\text { Lang, } 1965^{14}\end{array}$ & NA & NA & NA & At, Pacific \\
\hline $\begin{array}{l}\text { Stenhelia clavus } \\
\text { Wells and Rao, } 1987^{10,13}\end{array}$ & NA & NA & NA & NA, NWP \\
\hline $\begin{array}{l}\text { Typhlamphiascus lutincola } \\
\text { Soyer, } 1963^{29}\end{array}$ & NA & NA & NA & NA, NWP \\
\hline $\begin{array}{l}\text { Typhlamphiascus typhloides } \\
\text { (Sars G.0., 1911) }\end{array}$ & NA & NA & NA & WCP \\
\hline
\end{tabular}




\section{MONSTRILLIDAE}

\begin{tabular}{|c|c|c|c|c|}
\hline $\begin{array}{l}\text { Cymbasoma bullatus } \\
\text { (ScottA., 1909) }^{1}\end{array}$ & NA & NA & NA & NWP \\
\hline \multicolumn{5}{|l|}{ OITHONIDAE } \\
\hline 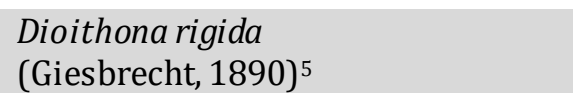 & Sac-spawner & Herbivorous & NA & NEA, ID, NP \\
\hline $\begin{array}{l}\text { Oithona aruensis } \\
\text { Früchtl, } 1923^{2,3,4,11,26}\end{array}$ & Sac-spawner & Omnivorous & NA & NWP \\
\hline $\begin{array}{l}\text { Oithona attenuata } \\
\text { Farran, } 1913^{1,2,3,4,5,20,27}\end{array}$ & Sac-spawner & Omnivorous & NA & SA, NEA, ID, NP \\
\hline $\begin{array}{l}\text { Oithona brevicornis } \\
\text { Giesbrecht, } 1891^{1,2,3,4,5,26,27}\end{array}$ & Sac-spawner & Omnivorous & NA & SEA, NEA, ID, NWP \\
\hline $\begin{array}{l}\text { Oithona decipiens } \\
\text { Farran, } 1913^{20}\end{array}$ & Sac-spawner & Omnivorous & Ambush & NA, ID, NP, SEP \\
\hline $\begin{array}{l}\text { Oithona dissimilis } \\
\text { Lindberg, } 1940^{2,3,4,5}\end{array}$ & Sac-spawner & Omnivorous & NA & ID, NWP \\
\hline $\begin{array}{l}\text { Oithona fallax } \\
\text { Farran, } 1913^{31}\end{array}$ & Sac-spawner & Omnivorous & NA & SEA, ID, NP, SEP \\
\hline $\begin{array}{l}\text { Oithona nana } \\
\text { Giesbrecht, } 1893^{9,11,20,27,31}\end{array}$ & Sac-spawner & Omnivorous & Predator & Atl, ID, Pacific \\
\hline $\begin{array}{l}\text { Oithona oculata } \\
\text { Farran, } 1913^{20,27}\end{array}$ & Sac-spawner & Omnivorous & NA & \\
\hline $\begin{array}{l}\text { Oithona plumifera } \\
\text { Baird, } 1843^{1,3,5,20,27}\end{array}$ & Sac-spawner & $\begin{array}{l}\text { Carnivorous- } \\
\text { omnivorous }\end{array}$ & Ambush & AA, Atl, ID, Pacific \\
\hline $\begin{array}{l}\text { Oithona rigida } \\
\text { Giesbrecht, } 18961,3,4,11,20,26,27,31\end{array}$ & Sac-spawner & Omnivorous & NA & \\
\hline $\begin{array}{l}\text { Oithona setigera } \\
\text { (Dana, } 1849)^{20}\end{array}$ & Sac-spawner & Omnivorous & Ambush & Atl, ID, Pacific \\
\hline $\begin{array}{l}\text { Oithona similis } \\
\text { Claus, } 1866^{27}\end{array}$ & Sac-spawner & Omnivorous & Ambush & AA, Pacific, ID \\
\hline $\begin{array}{l}\text { Oithona simplex } \\
\text { Farran, } 1913^{1,2,3,4,5,9,20,23,26,27}\end{array}$ & Sac-spawner & Omnivorous & NA & SEA, NEA, ID, NP \\
\hline \multicolumn{5}{|l|}{ ONCAEIDAE } \\
\hline $\begin{array}{l}\text { Oncaea clevei } \\
\text { Früchtl, 192313,4,5,27 }\end{array}$ & Sac-spawner & Omnivorous & NA & NEA, ID, NWP \\
\hline $\begin{array}{l}\text { Oncaea conifera } \\
\text { Giesbrecht, } 1891^{20,21,23}\end{array}$ & Sac-spawner & NA & NA & \\
\hline $\begin{array}{l}\text { Oncaea media } \\
\text { Giesbrecht, } 1891^{1,11,26,27,31}\end{array}$ & Sac-spawner & $\begin{array}{l}\text { Detritivorou- } \\
\text { omnivorous }\end{array}$ & Predator & Atl, ID, NP \\
\hline $\begin{array}{l}\text { Oncaea mediterranea } \\
\text { (Claus, } 1863)^{20}\end{array}$ & Sac-spawner & $\begin{array}{l}\text { Detritivorou- } \\
\text { omnivorous }\end{array}$ & Predator & SWP, ID, NEP, NWP \\
\hline $\begin{array}{l}\text { Oncaea paraclevei } \\
\text { Böttger-Schnack, } 2001^{27}\end{array}$ & Sac-spawner & Omnivorous & NA & ID \\
\hline $\begin{array}{l}\text { Oncaea scottodicarloi } \\
\text { Heron and Bradford-Grieve, 19951,27 }\end{array}$ & Sac-spawner & $\begin{array}{l}\text { Detritivorou- } \\
\text { herbivorous }\end{array}$ & NA & NEA, ID, NWP \\
\hline $\begin{array}{l}\text { Oncaea similis } \\
\text { Sars G.O., } 1918^{1}\end{array}$ & Sac-spawner & Omnivorous & NA & SWP, ID, WCP, NWP \\
\hline $\begin{array}{l}\text { Oncaea venusta } \\
\text { Philippi, } 1843^{1,11,27}\end{array}$ & Sac-spawner & $\begin{array}{l}\text { Detritivorou- } \\
\text { omnivorous }\end{array}$ & Predator & NP, ID, SEP, AA, A \\
\hline \multicolumn{5}{|l|}{ PARACALANIDAE } \\
\hline $\begin{array}{l}\text { Acrocalanus gibber } \\
\text { Giesbrecht, } 1888^{1,2,3,4,5,9,11,20,23,26,27,28}\end{array}$ & NA & Herbivorous & $\begin{array}{l}\text { Filter- } \\
\text { feeders }\end{array}$ & NA, ID, NP, SEP \\
\hline $\begin{array}{l}\text { Acrocalanus gracilis } \\
\text { Giesbrecht, } 1888^{1,3,4,9,11,20,23,26,27,28}\end{array}$ & NA & $\begin{array}{l}\text { Herbivorous- } \\
\text { omnivorous }\end{array}$ & $\begin{array}{l}\text { Filter- } \\
\text { feeders }\end{array}$ & ID, Pacific \\
\hline $\begin{array}{l}\text { Acrocalanus longicornis } \\
\text { Giesbrecht, } 1888^{1,9,26,27,28}\end{array}$ & NA & Herbivorous & $\begin{array}{l}\text { Filter- } \\
\text { feeders }\end{array}$ & ID, NA, NP, SEP \\
\hline
\end{tabular}




\begin{tabular}{|c|c|c|c|c|}
\hline $\begin{array}{l}\text { Acrocalanus monachus } \\
\text { Giesbrecht, } 1888^{5,11,27}\end{array}$ & NA & Herbivorous & $\begin{array}{l}\text { Filter- } \\
\text { feeders }\end{array}$ & ID, NP, SEP, NEA \\
\hline $\begin{array}{l}\text { Bestiolina similis } \\
\text { (Sewell, 1914) })^{1,2,3,4,5,9,11,20,23,27,28}\end{array}$ & Broadcaster & Herbivorous & NA & ID, NWP, SWA \\
\hline $\begin{array}{l}\text { Calocalanus pavo } \\
(\text { Dana, } 1852)^{27}\end{array}$ & Broadcaster & $\begin{array}{l}\text { Herbivorous- } \\
\text { omnivorous }\end{array}$ & $\begin{array}{l}\text { Filter- } \\
\text { feeders }\end{array}$ & ID, NA, NP, SEP \\
\hline $\begin{array}{l}\text { Calocalanus pavoninus } \\
\text { Farran, } 1936^{23}\end{array}$ & Broadcaster & $\begin{array}{l}\text { Herbivorous- } \\
\text { omnivorous }\end{array}$ & $\begin{array}{l}\text { Filter- } \\
\text { feeders }\end{array}$ & $\begin{array}{l}\text { SWP, SEP, ID, } \\
\text { WCP, NWP }\end{array}$ \\
\hline $\begin{array}{l}\text { Calocalanus styliformis } \\
\text { Giesbrecht, } 1888^{27,29}\end{array}$ & Broadcaster & Omnivorous & NA & $\begin{array}{l}\text { SWA, ID, NP, } \\
\text { NWA, SEP }\end{array}$ \\
\hline $\begin{array}{l}\text { Paracalanus aculeatus } \\
\text { Giesbrecht, } 1888^{1,2,3,4,5,11,20,23,26,27,28}\end{array}$ & Broadcaster & Omnivorous & $\begin{array}{l}\text { Filter- } \\
\text { feeders }\end{array}$ & Atl, ID, Pacific \\
\hline $\begin{array}{l}\text { Paracalanus crassirostris } \\
\text { Dahl F., } 18941,9,23,26\end{array}$ & Broadcaster & Omnivorous & NA & Atl, ID, NP \\
\hline $\begin{array}{l}\text { Paracalanus denudatus } \\
\text { Sewell, } 1929^{1,3,9,20,27,28}\end{array}$ & Broadcaster & $\begin{array}{l}\text { Herbivorous- } \\
\text { omnivorous }\end{array}$ & $\begin{array}{l}\text { Filter- } \\
\text { feeders }\end{array}$ & SEA, NEA, ID, NWP \\
\hline $\begin{array}{l}\text { Paracalanus elegans } \\
\text { (Andronov, 1972) 1,9,23,26,32 }\end{array}$ & Broadcaster & Omnivorous & NA & ID, WCP \\
\hline $\begin{array}{l}\text { Paracalanus indicus } \\
\text { Wolfenden, } 1905^{31}\end{array}$ & Broadcaster & Omnivorous & $\begin{array}{l}\text { Suspensio } \\
\mathrm{n} \text { feeders }\end{array}$ & Pacific, NEA, ID \\
\hline 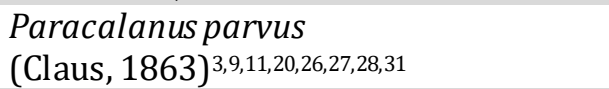 & Broadcaster & Herbivorous & $\begin{array}{l}\text { Filter- } \\
\text { feeders }\end{array}$ & ID, Atl, AA, Pacific \\
\hline $\begin{array}{l}\text { Parvocalanus crassirostris } \\
\text { (Dahl F., 1894) } \text { (2,3,4,5,20,27,28 }^{-1}\end{array}$ & Broadcaster & $\begin{array}{l}\text { Detritivorou- } \\
\text { omnivorous }\end{array}$ & $\begin{array}{l}\text { Filter- } \\
\text { feeders }\end{array}$ & Atl, ID, NP \\
\hline $\begin{array}{l}\text { Parvocalanus elegans } \\
\text { Andronov, } 1972^{2,3,4,20,28,31}\end{array}$ & Broadcaster & NA & NA & ID, NWP \\
\hline \multicolumn{5}{|l|}{ PARASTENHELLIDAE } \\
\hline $\begin{array}{l}\text { Parastenhelia hornelli } \\
\text { Thompson I.C. and Scott A., 190310,13 }\end{array}$ & NA & NA & NA & NA, SWP, NWP \\
\hline $\begin{array}{l}\text { Parastenhelia littoralis } \\
\text { (Sars G.O., 1911) }^{12,13}\end{array}$ & NA & NA & NA & NA, WCP \\
\hline \multicolumn{5}{|l|}{ PELTIDIIDAE } \\
\hline $\begin{array}{l}\text { Clytemnestra scutellata } \\
\text { Dana, } 18471,3,4,5,11,20,23,26,27\end{array}$ & NA & Herbivorous & NA & ID, SWA, NWP \\
\hline \multicolumn{5}{|l|}{ PHAENNIDAE } \\
\hline $\begin{array}{l}\text { Phaenna spinifera } \\
\text { Claus, } 1863^{1,11,31}\end{array}$ & Broadcaster & $\begin{array}{l}\text { Herbivorous- } \\
\text { omnivorous }\end{array}$ & Mixed & Atl, ID, NWP, SEP \\
\hline \multicolumn{5}{|l|}{ PONTELLIDAE } \\
\hline $\begin{array}{l}\text { Calanopia australica } \\
\text { Bayly and Greenwood, } 1996^{20}\end{array}$ & Broadcaster & Carnivorous & NA & ID \\
\hline $\begin{array}{l}\text { Calanopia aurivilli } \\
\text { Cleve, } 1901^{20}\end{array}$ & Broadcaster & Carnivorous & NA & ID, SWA \\
\hline $\begin{array}{l}\text { Calanopia elliptica } \\
\text { (Dana, 1849) }^{5,20,23,27,29}\end{array}$ & Broadcaster & Omnivorous & NA & NA, ID, NP \\
\hline $\begin{array}{l}\text { Calanopia minor } \\
\text { Scott A., } 1902^{1,23,27,29}\end{array}$ & Broadcaster & Carnivorous & NA & ID, NWP \\
\hline $\begin{array}{l}\text { Calanopia thompsoni } \\
\text { Scott A., 19093,4,5,20,23,27 }\end{array}$ & Broadcaster & Carnivorous & NA & ID, NWP \\
\hline $\begin{array}{l}\text { Labidocera acuta } \\
\text { (Dana, 1849) })^{1,3,5,11,20,26,27,29}\end{array}$ & Broadcaster & Carnivorous & NA & NEA, ID, NP, SEP \\
\hline $\begin{array}{l}\text { Labidocera bengalensis Krishnaswamy, } \\
1952^{5,20,27}\end{array}$ & Broadcaster & Carnivorous & NA & ID, SWP \\
\hline $\begin{array}{l}\text { Labidocera euchaeta } \\
\text { Giesbrecht, } 1889^{3,4,5,9,26,27}\end{array}$ & Broadcaster & Carnivorous & NA & ID, NP \\
\hline $\begin{array}{l}\text { Labidocera jaafari } \\
\text { Othman, } 1986^{3,4,5,25,26}\end{array}$ & Broadcaster & Carnivorous & NA & Malacca Straits \\
\hline
\end{tabular}


Labidocera javaensis

Mulyadi, $1997^{26}$

\section{Labidocera kroyeri \\ (Brady, 1883) $1,5,20,26,27$ \\ Labidocera minuta}

Giesbrecht, 188920,21,23,26,27,29

Labidocera pavo

Giesbrecht, $1889^{5}$

Labidocera pectinata

Thompson I.C. and ScottA., 19031,3,4,5,27

Labidocera rotundata

Mori, 192927

Pontelladanae

Giesbrecht, $1889^{3}$

Pontellafera

Dana, 18491,31

Pontellainvestigatoris

Sewell, $1912^{5}$

Pontella securifer

Brady, $1883^{5}$

Pontellina plumata

(Dana, 1849) ${ }^{1,27}$

Pontellopsis herdmani

Thompson I.C. and ScottA., $1903^{20}$

Pontellopsis krameri

(Gisbrecht, 1896) 3,20 $^{3,20}$

Pontellopsis macronyx

Scott A., 190920

Pontellopsis regalis

(Dana, 1849) ${ }^{26}$

Pontellopsis tenuicauda

(Giesbrecht, 1889) ${ }^{1,3}$

\section{PORCELLIDIIDAE}

Dilatatiocaudamedialis

Harris V.A., $2002^{15}$

Kensakiaparva

Harris V.A. and Iwasaki, 19976

Porcellidium aiiroa

(Harris V.A. and Iwasaki, 1997) ${ }^{15}$

Porcellidium brevicaudatum

Thompson I.C. and ScottA., $1903^{15}$

Porcellidium fimbriatum

Claus, 186312,13

Porcellidium poorei

Walker-Smith, $2001^{13}$

Porcellidium ofunatense

Harris V.A. and Iwasaki, $1996{ }^{15}$

Porcellidium yoroium

(Harris V.A. and Iwasaki, 1997) ${ }^{15}$

\section{PSEUDODIAPTOMIDAE}

Pseudodiaptomus annandalei

Sewell, 19193,4,18,19

Pseudodiaptomus aurivilli

Cleve, 190127,29

Pseudodiaptomus bowmani

Walter, 19841,3,4,5
Broadcaster

Carnivorous

NA

Carnivorous NA

Carnivorous NA

Carnivorous NA

Carnivorous NA

Carnivorous NA

NA

NA

NA

NA

NA

NA

NA

NA

NA

NA

NA

NA

NA

NA

NA

NA

NA

NA

NA

NA

NA
NA

NA

NA

WCP

NA

NA

NA

Carnivorousomnivorous

NA

NA

NA

NA

NA

NA

NA

NA

NA

NA

NA

NA

NA

NA

NA

NA

NA

NA

NA

Detritivorou-

herbivorous

NA

ID, NWP

Omnivorous

NA

Herbivorous NA
ID, NP

Java Seas,

The South China Sea, Malacca Straits

NEA, ID, NWP

NEA, ID, NP

NEA, ID, NWP

ID, South China Sea

ID, South China Sea

ID, NWP

ID

NA, ID, NWP, SEP

A, ID, Pacific

ID, South China Sea

ID, South China Sea

NA, ID, NWP, SEP

ID, NWP

ID, WCP 


\begin{tabular}{|c|c|c|c|c|}
\hline $\begin{array}{l}\text { Pseudodiaptomus clevei } \\
\text { Scott A., } 1909^{20}\end{array}$ & NA & Herbivorous & NA & ID \\
\hline $\begin{array}{l}\text { Pseudodiaptomus dauglishi } \\
\text { Sewell, } 1932^{9}\end{array}$ & NA & Herbivorous & NA & ID \\
\hline $\begin{array}{l}\text { Pseudodiaptomus incisus } \\
\text { Shen and Le, } 1963^{20}\end{array}$ & NA & Herbivorous & NA & WCP \\
\hline $\begin{array}{l}\text { Pseudodiaptomus thailandensis } \\
\text { (Walter, 1984) }^{3,4,5}\end{array}$ & NA & Herbivorous & NA & WCP \\
\hline $\begin{array}{l}\text { Pseudodiaptomus trihamatus } \\
\text { WrightS., 19373,4,19 }\end{array}$ & NA & Herbivorous & NA & WCP \\
\hline \multicolumn{5}{|l|}{ SAPPHIRINIDAE } \\
\hline $\begin{array}{l}\text { Copilialata } \\
\text { Dana, } 1849^{26}\end{array}$ & NA & Cornivorous & NA & NEA, NWP, SWP \\
\hline $\begin{array}{l}\text { Copilialongistylis } \\
\text { Mori, } 1932^{3}\end{array}$ & NA & Cornivorous & NA & ID, NWP, SEP \\
\hline $\begin{array}{l}\text { Copilia mirabilis } \\
\text { Dana, 184923,26,27 }\end{array}$ & Sac-spawner & Cornivorous & NA & $\begin{array}{l}\text { SEA, NEA, ID, } \\
\text { NP, SEP }\end{array}$ \\
\hline $\begin{array}{l}\text { Copilia mirabilis platyonyx } \\
\text { Paiva, } 1971^{1,3}\end{array}$ & Sac-spawner & Cornivorous & NA & ID, NWP \\
\hline $\begin{array}{l}\text { Copilia quadrata } \\
\text { Dana, } 1849^{27}\end{array}$ & Sac-spawner & Cornivorous & NA & SEA, NA, NP \\
\hline $\begin{array}{l}\text { Sapphirina agusta } \\
\text { Dana, } 1849^{27}\end{array}$ & Sac-spawner & Carnivorous & NA & $\begin{array}{l}\text { SEA, NA, ID, } \\
\text { NWP, SEP }\end{array}$ \\
\hline $\begin{array}{l}\text { Sapphirina gastrica } \\
\text { Giesbrecht, } 1891^{5,27}\end{array}$ & Sac-spawner & Carnivorous & NA & SEA, ID \\
\hline $\begin{array}{l}\text { Sapphirina metallina } \\
\text { Dana, } 1849^{27}\end{array}$ & Sac-spawner & Carnivorous & NA & AA, NA, ID, NP, SEP \\
\hline \multicolumn{5}{|l|}{ SCOLECITRICHIDAE } \\
\hline $\begin{array}{l}\text { Scolecithricella minor } \\
(\text { Brady, 1883) }\end{array}$ & NA & Detrivorous & NA & $\begin{array}{l}\text { AA, SWA, SWP, } \\
\text { SEP, A, ID }\end{array}$ \\
\hline $\begin{array}{l}\text { Scolecithrix nicobarica } \\
\text { Sewell, } 1929^{1}\end{array}$ & NA & NA & NA & SEA, ID, NP \\
\hline \multicolumn{5}{|l|}{ SUBEUCALANIDAE } \\
\hline $\begin{array}{l}\text { Subeucalanus subcrassus } \\
\text { (Giesbrecht, 1888) } 5,11,20,23\end{array}$ & Broadcaster & Herbivorous & NA & NA, ID, NP, SEP \\
\hline $\begin{array}{l}\text { Subeucalanus subtenuis } \\
\text { (Giesbrecht, 1888) }\end{array}$ & Broadcaster & Herbivorous & $\begin{array}{l}\text { Filter- } \\
\text { feeders }\end{array}$ & NWA, ID, NP, SEP \\
\hline \multicolumn{5}{|l|}{ TACHIDIIDAE } \\
\hline $\begin{array}{l}\text { Euterpina acutifrons } \\
\text { (Dana, 1848) })^{1,2,3,4,5,9,11,20,21,23,26,27,31}\end{array}$ & Sac-spawner & $\begin{array}{l}\text { Detrivorous- } \\
\text { omnivorous }\end{array}$ & Grazers & Atl, Pacific, ID \\
\hline $\begin{array}{l}\text { Tachidius discipes } \\
\text { Giesbrecht, } 1881^{32}\end{array}$ & NA & NA & NA & WCP, ID, NEP, NEA \\
\hline \multicolumn{5}{|l|}{ TEMORIDAE } \\
\hline $\begin{array}{l}\text { Temora discaudata } \\
\text { Giesbrecht, } 18891,3,4,5,11,20,21,23,26,27\end{array}$ & Broadcaster & $\begin{array}{l}\text { Herbivorous- } \\
\text { omnivorous }\end{array}$ & $\begin{array}{l}\text { Filter- } \\
\text { feeders }\end{array}$ & Atl, Pacific, ID \\
\hline $\begin{array}{l}\text { Temora stylifera } \\
\text { (Dana, 1849) } 1,11,26,27,31\end{array}$ & Broadcaster & $\begin{array}{l}\text { Herbivorous- } \\
\text { omnivorous }\end{array}$ & $\begin{array}{l}\text { Filter- } \\
\text { feeders }\end{array}$ & Atl, Pacific, ID \\
\hline $\begin{array}{l}\text { Temora turbinata } \\
\text { (Dana, 1849) }^{1,3,4,5,20,23,26,27,29,31}\end{array}$ & Broadcaster & $\begin{array}{l}\text { Herbivorous- } \\
\text { omnivorous }\end{array}$ & NA & Pacific, NA, ID \\
\hline \multicolumn{5}{|l|}{ TISBIDAE } \\
\hline $\begin{array}{l}\text { Tisbe bermudensis } \\
\text { Willey, } 1930^{13}\end{array}$ & NA & NA & NA & Atl, NWP \\
\hline \multicolumn{5}{|l|}{ TETRAGONICIPITIDAE } \\
\hline $\begin{array}{l}\text { Phyllopodopsyllusborutskyi } \\
\text { Lang, 196510,13 }\end{array}$ & NA & NA & NA & NWP \\
\hline
\end{tabular}




\section{THALESTRIDAE}

\begin{tabular}{|c|c|c|c|c|}
\hline $\begin{array}{l}\text { Eudactylopus andrewi } \\
\text { Sewell, } 1940^{7,12,13}\end{array}$ & NA & NA & NA & NA, ID, WCP \\
\hline $\begin{array}{l}\text { Eudactylopus fasciatus } \\
\text { Sewell, } 1940^{12,13}\end{array}$ & NA & NA & NA & NA, ID, WCP \\
\hline $\begin{array}{l}\text { Eudactylopus latipes } \\
\text { (Scott T., 1893) }\end{array}$ & NA & NA & NA & NA, ID, WCP \\
\hline $\begin{array}{l}\text { Phyllothalestris mysis } \\
\text { (Claus, 1863) }\end{array}$ & NA & NA & NA & NA, ID, WCP \\
\hline $\begin{array}{l}\text { Phyllothalestris sarsi } \\
\text { Sewell, 1904 } 13\end{array}$ & NA & NA & NA & ID, WCP \\
\hline $\begin{array}{l}\text { Rhynchothalestris rufocincta } \\
\text { (Brady, 1880) }{ }^{13}\end{array}$ & NA & NA & NA & Atl, NWP \\
\hline \multicolumn{5}{|l|}{ TORTANIDAE } \\
\hline 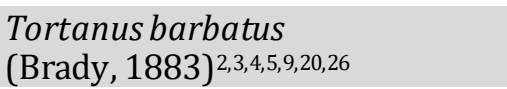 & NA & Carnivorous & NA & ID, NWP \\
\hline $\begin{array}{l}\text { Tortanus forcipatus } \\
\text { (Giesbrecht, 1889) }{ }^{1,2,3,4,4,9,20,26,27,28}\end{array}$ & NA & Carnivorous & NA & ID, NWP \\
\hline $\begin{array}{l}\text { Tortanus gracilis } \\
\text { (Brady, 1883) }^{20,23,26,27}\end{array}$ & NA & Carnivorous & NA & ID, NWP \\
\hline $\begin{array}{l}\text { Tortanus longipes } \\
\text { Brodsky, } 1948^{23}\end{array}$ & NA & NA & NA & NWP \\
\hline
\end{tabular}

General biogeography: NWP= NW Pacific; NEA= NE Atlantic; NA= North Atlantic; NP=North Pacific; SEA=SE Atlantic; SWA=SW Atlantic; SEP=SE Pacific; SWP=SW Pacific; ID=Indian Ocean; WCP=Western Central Pacific; WCA=Western Central Atlantic; Pacific= whole Pacific; Atl= whole Atlantic; A=Artic; AA=Antarctic; NA=notavailable.

$1=$ Chew et al. (2008)

$2=$ Chew et al. (2011)

$3=$ Chew (2012)

$4=$ Chew et al. (2015)

$5=$ Chew et al. (2016)

$6=$ Harris et al. (2009)

$7=$ Ishak et al. (2005)

$8=$ Ishak et al. (2009)

$9=$ Ismail et al. (2012)

$\begin{array}{ll}\text { 10=Kassim etal. (2006) } & \text { 19=Liu et al. (2015) } \\ \text { 11=Kassim etal. (2008) } & 20=\text { Metillo etal. (2019) } \\ \text { 12=Kassim etal. (2010) } & 21=\text { Nakajima et al. (2009) } \\ \text { 13=Kassim etal. (2011) } & 22=\text { Nakajima et al. (2013) } \\ \text { 14=Kassim etal. (2012) } & 23=\text { Nakajima et al. (2015) } \\ \text { 15=Kassim etal. (2013) } & 24=\text { Ohtsuka et al. (2020) } \\ \text { 16=Kassim etal. (2018) } & 25=\text { Othman (1986) } \\ \text { 17=Kassim etal. (2019) } & 26=\text { Peralta et al. (2015) } \\ \text { 18=Lehette et al. (2016) } & 27=\text { Rezai et al. (2004) }\end{array}$

$10=$ Kassim etal. (2006)

$11=$ Kassim etal. (2008)

$13=$ Kassimetal. (2011)

$14=$ Kassim et al. (2012)

15=Kassim etal. (2013)

17=Kassinetal. (2019)

18=Lehette et al. (2016)
28=Rezai et al. (2005)

$29=$ Sham (2019)

$30=$ Sham et al. (2020)

$31=$ Shuaib et al. (2019)

$32=$ Somerfield et al. (1998)

$33=W$. Rasdi etal. (2018)

$34=Y o s h i d a$ etal. (2006)

$35=$ Yoshida etal. (2012)

$36=$ Zuraire et al. (2018) 


\section{Result}

Table 1 shows the list of copepods found along the coast of Peninsular Malaysia. A total of 235 species were recorded, belonging to 89 genera, 44 families and 6 orders. One hundred and ninety-four species were recorded on the West Coast, an increase of about 38\% (75 species) over previous studies by Rezai et al. (2004, 2005, 2009), with Chew et al. (2008, 2011, 2012, 2015, 2016) focusing on copepods distribution in Malacca Strait's estuaries. In contrast, the East Coast contributed approximately $43 \%$ (53 species) to the increment of species discovered, and 131 species were recorded (Metillo et al., 2019).

Calanoida is the most diverse, $43 \%$ (Figure 2) with 101 species (30 genera), followed by Harpacticoida (29\%) with 70 species ( 45 genera). Moreover, Cyclopoida (25\%) has 61 species (8 genera). Canuelloida, Siphonostomatoida and Monstrilloida are the least diverse orders, each with 1 genus and 1 species, respectively.

Overall, there are four dominant genera in the list: Corycaeus with 19 species, Oithona (13 species),
Labidocera (10 species), Oncaea (8 species) and Pseudodiaptomus (8 species). Additionally, 6 distinct species are dominant throughout the coast of Peninsular Malaysia: Acartia erythraea (Giesbrecht, 1998), Bestiolina similis (Sewell, 1914), Euterpina acutifrons (Dana, 1848), Microsetella norvegica (Boeck, 1865), Paracalanus aculeatus (Giesbrecht, 1888) and Oithona simplex (Farran, 1913).

Interestingly, 3 species on this checklist can be categorised as endemic: 1 species was found on the West Coast, while 2 species were discovered on the East Coast. All the 3 species belonged to different orders: Kensakia parva (Harris V.A and Iwasaki, 1997) belongs to the order of Harpacticoida, and Brachiella malayensis (Ohtsuka, Piasecki, Ismail and Kamarudin, 2020) is from the order Siphonostomatoida which was most recently found on the East Coast of Peninsular Malaysia (Ohtsuka et al., 2020). Meanwhile, Labidocera jaafari Othman, 1986, was found in the Straits of Malacca, becoming the first endemic species reported in Peninsular Malaysia (Othman, 1986). In addition, 34 taxa of commonly found copepods from the coast of Peninsular Malaysia were photo-documented (Figure 3).

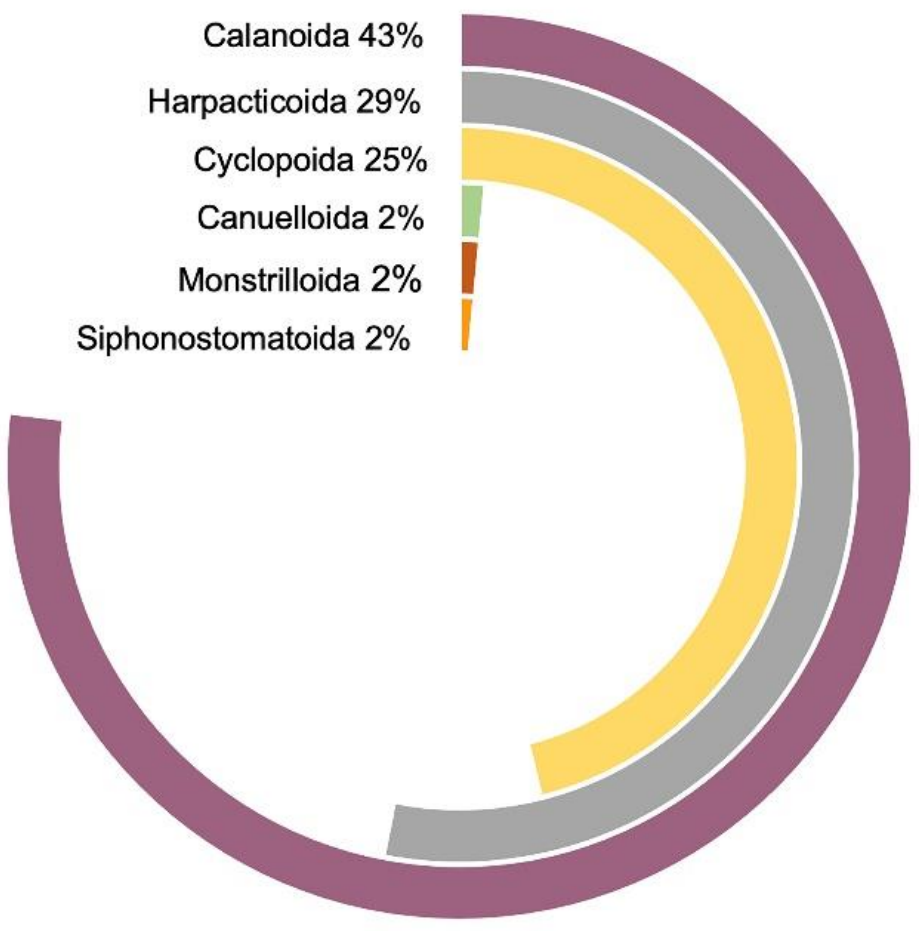

Figure 2. The percentage of copepod orders presented in the checklist. 

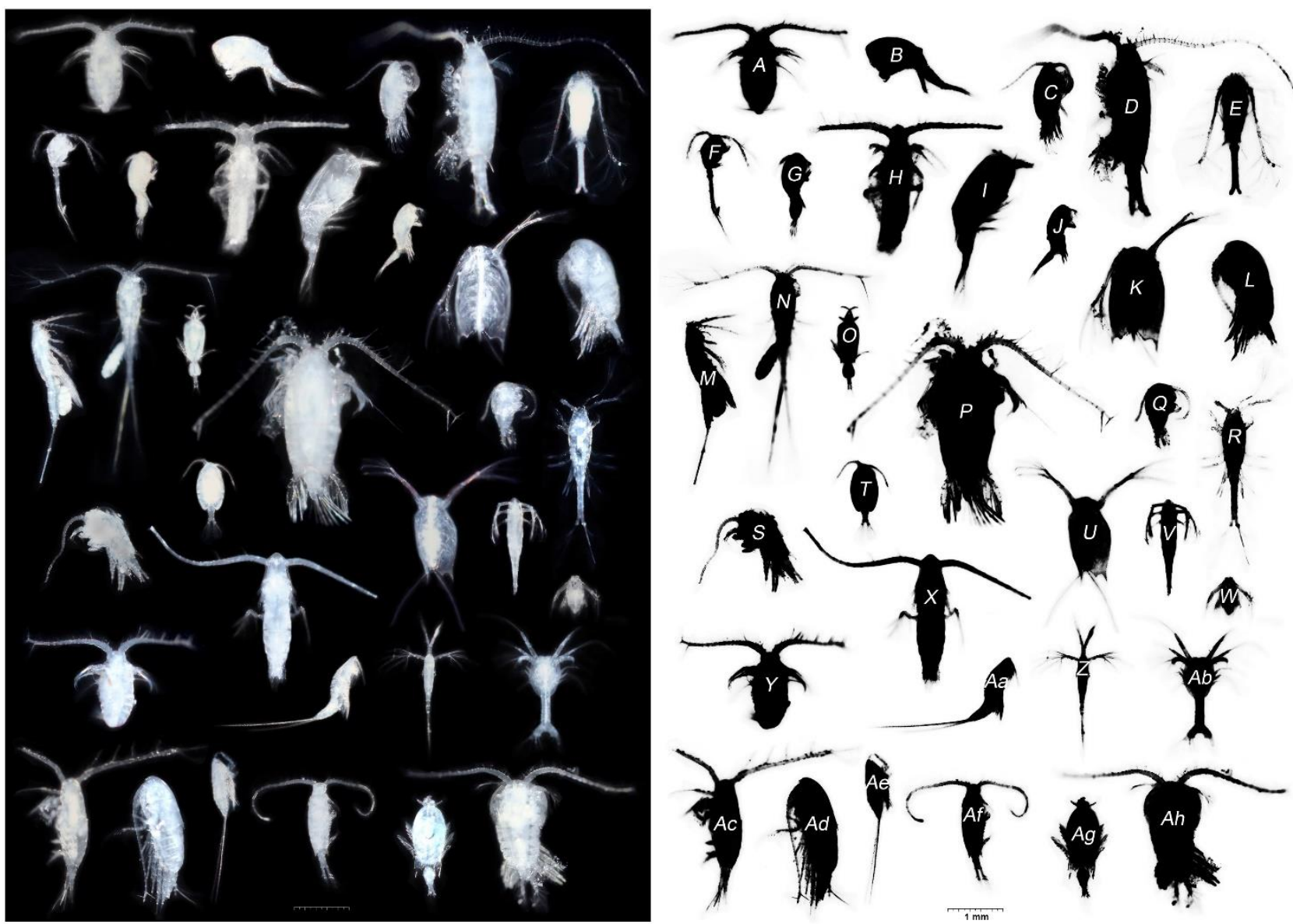

Figure 3. Microphotograph of one of the most abundant copepods along the coast of Peninsular Malaysia. ACandacia sp., B-Oncaea sp., C-Phaenna sp., D-Calanopia sp., E-Oithona sp., F-Oithona sp., G-Oncaea sp., HSubeucalanus sp., I-Corycaeus sp., J-Oncaea sp., K-Isopod, L-Paracalanus sp., M-Macrosetella sp., N-Oithona sp., OCorycaeus sp., P-Centropages sp., Q-Temora sp., R-Oithona sp., S-Centropages sp., T-Metacalanus sp., U-Isopod. VCopepodites, W-Nauplius, X-Subeucalanus sp., Y-Candacia sp., Z-Copepodites, Aa-Microsetella sp., Ab-Panaeid protozoa, Ac-Acartia sp., Ad-Paracalanus sp., Ae-Microsetella sp., Af-Acartia sp., Ag-Corycaeus sp., Ah-Centropages sp. 


\section{Discussion}

Rezai et al. (2004) identified a total of 117 species of copepods from 37 genera and 25 families. Oithona simplex, Euterpina acutifrons and Paracalanus parvus (Claus, 1863) were the dominant species. Additionally, Yoshida et al. (2006) reported 6 dominant genera of copepods in the Straits of Malacca: Acartia, Acrocalanus, Corycaeus, Euterpina, Paracalanus and Oithona. This discovery was supported by the identification of 71 species of copepods by Chew et al. (2008). There were 7 dominant species of copepods, namely Corycaeus andrewsi (Farran, 1911), E. acutifrons, M. norvegica, Oithona attenuate (Farran, 1913), Oithona brevicornis (Giesbrecht, 1891), Oncaea clevei (Früchtl, 1923) and Parvocalanus crassirostris (Dahl F., 1894). Compared to Rezai et al. (2004), this current study lacked 46 species but discovered 2 new families (Phaenidae and Montrilloidae).

Furthermore, Acrocalanus, Bestiolina, Paracalanus and Oithona were the dominant copepods identified among 48 species (33 genera and 23 families) of the East Coast zooplankton recorded (Nakajima et al., 2015). Moreover, Metillo et al. (2019) stated that Paracalanus elegans (Andronov, 1972) and O. simplex were the dominant species among 69 copepod species recorded. Kassim et al. (2006) conducted an ecological study and documented a total of 12 species belonging to 8 families and 10 genera.

This reflects a dearth of research on the distribution of copepods on the East Coast. South China Sea is estimated to house one-third of the world's marine biodiversity, making it a critical ecosystem (Yoshida et al., 2012). If not thoroughly explored, many species will stay undiscovered and remain unknown.

\section{Conclusion}

While extensive databases for zooplankton are being developed with more precise taxonomy and geographical distributions, the knowledge of marine copepods continues to have significant gaps due to the remoteness of some coastal states and the technical difficulty of studying copepods fauna in this region. Further field studies should be conducted in the area with more intensive samplings in a comprehensive bathymetric range.

This checklist is crucial because it establishes a foundation for baseline data for further studies. It provides information on species distribution, including the widespread species and possible invasive or endemic species. When the distribution of species is not adequately researched in a high diversity area, a lack of taxonomists becomes an issue. The species occurrence is not well documented.
In light of the effects of climate change on biodiversity, we believe that this checklist wouldserve as a reference for taxonomists, ecologists and other researchers, as well as for future follow-up studies. particularly for substantiating the distribution of climatically-significant species.

\section{Acknowledgement}

This study was conducted as part of several years of annual monitoring within Sultan Iskandar Marine Park (SIMP), Johor, with funding provided by the Ministry of Education (Malaysia) under the Fundamental Research Grant Scheme (FRGS/1/2019/WAB13/UKM/02/3). We would like to convey our gratitude to the Department of Fisheries (Malaysia) and the communities of the SIMP for their kind and generous support during our expeditions across the islands.

\section{References}

Alcaraz, M. \& Calbet, A. (2003). Zooplankton ecology. In: Marine Ecology (M. C. Duarte, eds.), pp 295-318. EOLSS Publications, Paris, France.

Araujo, A. V., Dias, C. O. \& Bonecker, S. L. C. (2020). Diversity and functional groups of copepods as a tool for interpreting trophic relationships and ecosystem functioning in estuaries. Marine Environmental Research 162,105-190. DOI https://doi.org/10.1016/j.marenvres.2020.105190

Benedetti, F., Gasparini, S. \& Ayata, S.D. (2016). Identifying copepod functional groups from species functional traits. Journal of Plankton $\begin{array}{lll}\text { Research 38(1), 159-166. DOE } & \text { 3 }\end{array}$ https: //doi.org/10.1093/plankt/fbv096

Benedetti, F., Vogt, M., Righetti, D., Guilhaumon, F. \& Ayata, S.D. (2018). Do functional groups of planktonic copepods differ in their ecological niches? Journal of Biogeography 45(3), 604-616. DO I: https://doi.org/10.1111/jbi.13166

Biodiversity of Marine Planktonic Copepods (morphology, geographical distribution and biological data. Marine Planktonic Copepod. Available online: https://copepodes.obsbanyuls.fr/en/index.php (accessed on November 2020).

Boesch, D. F. (1977). A new look at zonation of benthos along an estuarine gradient. In: Ecology of the Marine Benthos (B. C. Coull, ed.), pp 245-266. University of South Carolina Press, Columbia.

Campos, C. C., Garcia, T. M., Neumann-Leitão, S. \& Soares, M. O. (2017). Ecological indicators and functional groups of copepod assemblages. Ecological Indicators 83, 416-426. DOI https://doi.org/10.1016/j.ecolind.2017.08.018.

Carola, M. (1994). Checklist of the marine planktonic copeoda of Southern Africa and their worldwide geographic distribution. South African Journal of Marine Science 14(1), 225-253. DOI: https: //d oi.org/10.2989/025776194784287058.

Chew, L.L. \& Chong, V. C. (2011). Copepod community structure and abundance in a tropical mangrove estuary, with comparisons to coastal waters. Hydrobiologia 666(1), 127-143. DOI: https://doi.org/10.1007/s10750-010-0092-3.

Chew, L.L. \& Chong, V. C. (2016). Response of marine copepods to a changing tropical environment: winners, losers and implications. PeerJ 4,1-31, DOI: https://doi.org/10.7717/peerj.2052/supp-2. 
Chew, L.L., Chong, V. C. \& Ooi, A. (2008). Zooplankton of the Straits of Malacca, with emphasis on copepods and fish larvae in the vicinities of Jarak, Perak and Sembilan Islands. Malaysian Journal of Science 27,83-103.

Chew, L.L., Chong, V. C., Ooi, A. L. \& Sasekumar, A. (2015). Vertical migration and positioning behavior of copepods in a mangrove estuary: interactions between tidal, diel light and lunar cycles. Estuarine, Coastal and Shelf Science 152,142-152. DOI: https://doi.org/10.1016/j.ecss.2014.11.011

Chew, L. L. (2012). Dynamics and trophic role of zooplankton community in the Matang mangrove estuaries and adjacent coastal waters (Peninsular Malaysia), with special emphasis on copepods. Universiti Malaya, 308 pp. Universiti Malaya, Malaysia.

Dev Roy, M. \& Venkataraman, K. (2018). Catalogue on copepod fauna of India. part 2. cyclopoida and harpacticoida (arthropoda : crustacea). Journal of Environment and Sociobiology 15(2), 109-194.

Goswami, P. (2015). Studies on eutrophication, ocean acidification and trace metal pollution: effects on plankton communities from Southeast Coast of India. University of Madras, $95 \mathrm{pp}$. University of Madras, India.

Grindley, J. R. (1984). The zooplankton of mangrove stuaries. In: Hydrobiology of the Mangal (F. D. Por, \& I. Dor, eds.), pp 79-88. Dr W. Junk Publisher, Netherlands.

Harris, V. A. \& Iwasaki, N. (2009). Two new species of Kensakia belonging to the family porcellidiidae (crustacea, copepoda, harpacticoida) from Japan and Malaysia. Bulletin of the National Museum of Nature and Science. Series A, Zoology 35(3), 157-165.

Heip, C. H. R., Goosen, N. K., Herman, P. M. J., Kromkamp, J., Middelburg, J. J. \& Soetaert, K. (1995). Production and consumption of biological particles in temperate tidal estuaries. Oceanography and Marine Biology 33, 1-149.

Heinle, D. R. (2013). Zooplankton. In: Functional adaptation of marine organisms (F. J. Vernberg, \& W. B. Vernberg, eds.). pp 85-136. Academic Press, Inc., London.

Hicks, G. R. F. (1980). Structure of phytal harpacticoid copepod assemblages and the influence of habitat complexity and turbidity. Journal of Experimental Marine Biology and Ecology 44(2), 157-192. DOI: https://doi.org/10.1016/0022-0981(80)90151-3

Hopcroft, R. R. \& Roff, J. C. (1996). Zooplankton growth rates: diel egg production in the copepods Oithona, Euterpina and Corycaeus from tropical waters. Journal of Plankton Research 18(5), 789-803. DOI: https://doi.org/10.1093/plankt/18.5.789

Ishak, N. \& Kassim, Z. (2005). Report on some phytal harpacticoid copepods from Terengganu Coast. Proceedings of the KUSTEM 4th Annual Seminar pp 392-396.

Ishak, N. \& Kassim, Z. (2009). Taxonomy of a benthic copepod, Metamphiascopsis Hirsutus (Thompson \& A. Scott, 1903) (harpacticoida: Diosaccidae) from Bidong Island, Malaysia. Malaysian Journal of Microscopy 5, 36-41.

Ismail, J., Wan Omar, W. M., Mansor, M., Kamal, A. H. \& Amin, S. M. N. (2012). Spatial distribution of copepods along the salinity gradient of Perai River estuary, Penang, Malaysia. Pakistan Journal of Biological Sciences 15, 647-652. DOI: https://dx.doi.org/10.3923/pjbs.2012.647.652

Johan, I., Abu Hena, M. K., Idris, M. H. \& Arshad, A. (2013).Taxonomic composition and abundance of zooplankton copepoda in the coastal waters of Bintulu, Sarawak, Malaysia. Journal of Fisheries and Aquatic $\begin{array}{llll}\text { Science } & 8(3), & 472-479 . & \text { DOI: }\end{array}$ https://dx.doi.org/10.3923/jfas.2013.472.479
Kassim, Z., Abdul Kadar, N. \& Ishak, N. (2010). Species assemblages of benthic harpacticoid copepods on tide rock pool seaweeds of Pulau Besar, Melaka, Malaysia. Journal of Tropical Biology and Conservation 7, 1-10.

Kassim, Z., Hasnan, H., Zainal, S. \& Ishak, N. (2018). Report on five species of harpacticoid copepods from vegetative area of Sungai Pulai, Johor. Malaysian Journal of Fundamental and Applied Sciences 14, 284-288. DOI: https://doi.org/10.11113/mjfas.v14n2.1095

Kassim, Z., Huda, A., Ahmad, Z., Hanim, H., Azrina, S. \& Ishak, N. (2019). New record of harpacticoid (copepoda: harpacticoida) from Tioman Archipelago, Pahang, Peninsular Malaysia. Malayan Nature Journal 71, 3-15.

Kassim, Z., Ibrahim, B., John, A. \& Yunus, K. (2012). Generation time of some marine harpacticoid species in laboratory condition. Journal of $\begin{array}{llll}\text { Biological Sciences } & 12(8), & 1-5 . & \text { DOI: }\end{array}$ http://dx.doi.org/10.3923/jbs2012

Kassim, Z., Jefri, J. \& Hasimah. (2013). Fauna of porcellidiidae (copepoda: harpacticoida) from Sungai Pulai, Malaysia. Malayan Nature Journal 64, 159-167.

Kassim, Z., Kasmaruddin, K., Ishak, N., Mr, R., H, N. \& Yunus, K. (2011). Biodiversity of selected marine arthropoda in Malaysia. In: Malaysia's Marine Biodiversity: Inventory and Current Status (I. Kamaruddin, C. A. R. Mohamed, M. J. Rozaimi, et al., eds.), pp 99-108 Department of Marine Park Malaysia, Putrajaya, Malaysia.

Kassim, Z., Md Amin, R. \& Iwasaki, N. (2006). Distribution of some species of harpacticoid copepods in East Coast of Peninsular Malaysia. Coastal Marine Science 30 (1), 140-145.

Kassim, Z., Sulong, I., Lokman, M., Kamaruzaman, H. \& Baba, M. (2008). Species composition and abundance of planktonic copepods in Pahang Estuaries, Malaysia. Journal of Sustainability Science and Management 3(1), 11-22.

Kiørboe, T. (2011). How zooplankton feed: mechanisms, traits and trade-offs. Biological Reviews 86, 311-339. DOI https://doi.org/10.1111/j.1469-185x.2010.00148.x

Kiørboe, T. \& Sabatini, M. (1994). Repreductive and life cycle strategies in egg-carrying cyclopoid and free-spawning calanoid copepod. Journal of Plankton Research 16(10), 1353-1366. DOI http://dx.doi.org/10.1093/plankt/16.10.1353

Lehette, P., Ting, S. M., Chew, L.-L. \& Chong, V. C. (2016). Respiration rates of the copepod Pseudodiaptomus Annandalei in tropical waters: beyond the thermal optimum. Journal of Plankton Research 38(3), 456-467. DOI: https://doi.org/10.1093/plankt/fbv119

Liu, W., Tanimura, A., Yamada, K., Toyohara, H., Chew, L.-L., Hanamura, Y., Okutsu, T. \& Tanaka, K. (2015). Occurrence of cellulose activities in planktonic crustaceans inhabiting mangrove areas in Malaysia. Japan Agricultural Research Quarterly 49, 293-299. DO I http://dx.doi.org/10.6090/jarq.49.293

Metillo, E. B., Nishikawa, J., Ross, O. B. H., Yoshida, T., Md. Yusoff, F., Kuppan, P., Ohtsuka, S., Mulyadi, Sekiguchi, H., Toda, T. \& Nishida, S. (2019). Diel patterns of zooplankton community structure in nearshore waters of different substrates off Tinggi and Sibu Islands, Malaysia, with special reference to copepods. Aquatic Ecosystem Health \& Management 22(1), 86-102. DOI https://doi.org/10.1080/14634988.2018.1505139

Motoda, S. (1963). Corycaeus and Farranula (copepoda, cyclopoida) in Hawaiian waters. Publication of the Seto Marine Biological Laboratory 11(2), 209-262.

Mulyadi, H., Basit, A., Ikhsani, I. \& Abdul, M. (2018). Species richness abundance and vertical distribution of copepods in Banda Sea, Indonesia. E3S Web of Conferences 47, 1-9. DOI https://doi.org/10.1051/e3sconf/20184703004 
Nakajima, R., Yoshida, T., Ross, O. \& Toda, T. (2009). Diel variation of zooplankton in the tropical coral-reef water of Tioman Island, Malaysia. Aquatic Ecology 43, 965-975. DOI: http://dx.doi.org/10.1007/s10452-008-9208-5

Nakajima, R., Yoshida, T., Ross, O. \& Toda, T. (2013). First record of a blue-pigmented acartiid copepod in the tropical coral reef waters of Malaysia. Galaxea, Journal of Coral Reef Studies 15, 27-28. DOI: http://dx.doi.org/10.3755/galaxea.15.27

Nakajima, R., Yoshida, T., Ross, O. \& Toda, T. (2014). Biomass and estimated production rates of metazoan zooplankton community in a tropical coral reef of Malaysia. Marine Ecology 35, 112-131. DOI: http://dx.doi.org/10.1111/maec.12062

Nakajima, R., Yoshida, T., Ross, O. \& Toda, T. (2015). Monsoonal changes in the planktonic copepod community structure in a tropical coral-reef at Tioman Island, Malaysia. Regional Studies in Marine Science 2, 19-26. DOI: https://doi.org/10.1016/j.rsma.2015.08.016

Nishida, S. \& Nishikawa, J. (2011). Biodiversity of Marine Zooplankton in Southeast Asia (Project-3: Plankton Group). In: Coastal Marine Science in Southeast Asia: Synthesis Report of the Core University Program of the Japan Society for the Promotion of Science: Coastal Marine Science (2001-2010) (S. Nishida, M. D. Fortes, \& N. Miyazaki, eds.), pp 59-71. TERRAPUB, Tokyo, Japan.

Ohtsuka, S., Piasecki, W., Ismail, N. \& Kamarudin, A. S. (2020). A new Species of Brachiella (copepoda, siphonostomatoida, lernaeopodidae) from Peninsular Malaysia, with relegation of two genera Charopinopsis and Eobrachiella to junior synonyms of Brachiella. Parasite 27(40), 1776-1042. DOI: https://doi.org/10.1051/parasite/2020038

Othman, B. H. R. (1986). A new species of Labidocera (copepoda, calanoida) from Peninsular Malaysia. Malayan Nature Journal 39, 193201.

Othman, B. H. R. (1988). Pelagic copepod of the Economic Exclusive Zone Area in South China Sea. Thirty Years of Marine Science Research and Development. Proceedings of the 11th Annual Seminar, Malaysian Society of Marine Sciences, 26 March 1988, pp 8-14.

Peralta, H. M. \& Yusoff, F. (2015). Status of planktonic copepod diversity in the Merambong Seagrass Meadow, Johor, Peninsular Malaysia. International Journal of Ecosystem 5(2), 39-43. DOI: http://dx.doi.org/10.5923/j.ije.20150502.01

Por, F. (1964). A Study of the levantine and pontic harpacticoida (crustacea, copepoda). Zoologische Verhandelingen 64, 1-128.

Rezai, H., Arshad, A. B., Kawamura, A. \& Nishida, S. (2004). Spatial and temporal distribution of copepods in the Straits of Malacca. Zoological Studies 43(2), 486-497.

Rezai, H., Yusoff, F. M., Arshad, A. \& Othman, B. H. R. (2005). Spatial and temporal variations in calanoid copepod distribution in the Straits of Malacca. Hydrobiologia 537, 157-167. DOI: https://doi.org/10.1007/s10750-004-2792-z

Rezai, H., Yusoff, F. M., Arshad, A. \& Othman, B. H. R. (2009). Abundance and composition of zooplankton in the Straits of Malacca. Aquatic Ecosystem Health and Management 12(3), 264-270. DOI: https://doi.org/10.1080/14634980903149977

Sarkas, S. K. (1983). Distribution of Estuarine Epipelagic Zooplankton in and around Sagar Island, West Bengal, India. University of Calcutta, $261 \mathrm{pp}$. University of Calcutta, India.

Sewell, R. B. S. (1933). Notes on a small collection of marine copepoda from the Malay States. Bulletin Raffles Museum 8, 25-31.

Sham, A. (2019). New record of miraciidae (copepoda: harpacticoida) from Tioman Waters. International Journal of Recent Technology and Engineering (IJRTE) 8(1S), 351-358.
Sham, A., Kassim, Z., Ahmad, Z. \& Ismail, A. (2020). New report of Laophonte Cornuta Philippi,1840 (copepoda: harpacticoida) from the coral area, north of Tioman Island, South China Sea. Malaysian Journal of Fundamental and Applied Sciences 16(2), 232-235. DOI https://doi.org/10.11113/mjfas.v16n2.1438

Shuaib, N., Mohamed, M., Peralta, H. M., Rusiman, M. \& Sanusi, S. (2018). Preliminary observations of zooplankton in Pulau Tinggi. International Journal of Engineering and Technology 7(4.30), 94-95. DOI: http://dx.doi.org/10.14419/ijet.v7i4.30.22026

Shuaib, N., Mohammad, M., Monica Matias-Peralta, H., Saifullah Rusiman, M. \& Babaji Sanusi, S. (2019).Copepods status in seagrass area of Pulau Tinggi Marine Park, Johor, Malaysia. IOP Conference Series: Earth and Environmental Science 269, 1-5.

Somerfield, P., Gee, J. M. \& Aryuthaka, C. (1998). Meiofaunal communities in a Malaysian mangrove forest. Journal of the Marine Biological Association of the United Kingdom 78(3), 717-732. DOI http://dx.doi.org/10.1017/S0025315400044738

Spooner, G. M. \& Moore, H. B. (1940). The ecology of the Tamar Estuary VI. an account of the macrofauna of the intertidal muds. Journal of the Marine Biological Association of the United Kingdom 24(1), 283-330. DOI: https://doi.org/10.1017/S0025315400054588

Thistle, D., Carman K. R., Sedlacek L., Brewer P. G., Fleeger J. W. \& P., B. J. (2005). Deep-ocean, sediment-dwelling animals are sensitive to sequestered $\mathrm{CO}_{2}$. Marine Ecology Progress Series 289, 1-4. DOI http://dx.doi.org/10.3354/meps289001

Uye, S. \& Kayano, Y. (1994). Predatory feeding behaviour of Tortanus (copepoda: calanoida): life stage differences and the predation impact on small planktonic crustaceans. Journal of Crustacean Biology 14(3), 473-483, DOI: https://doi.org/10.1163/193724094X00 056.

W.Rasdi, N., Qin, J., Yeong, Y. S., Kassim, Z., Ikhwanuddin, M., Yu soff, F., Suhaimi, H., Yuslan, A. \& Omar, S. (2018). Effect of mono and binary diets on growth and reproduction of cyclopoid copepod. AACL Bioflux 11(5), 1658-1671.

Wickstead, J. H. (1961). A Qualitative and Quantitative Study of Some Indo-West-Pacific Plankton $\left(\mathbf{1 6}^{\text {th }}\right)$. Colonial Office Fisheries Publication, 200 pp. Colonial Office Fisheries Publication, Her Majesty's Stationery Office, London.

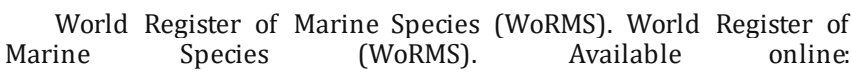
http://www.marinespecies.org at VLIZ (accessed on November 2020).

Yamazi, I. (1958). Preliminary checklist of planktonic organisms found in Tanabe Bay and its environs. Publication of the Seto Marine Biological Laboratory $7, \quad 111-163 . \quad$ DOI: https://doi.org/10.5134/174597

Yoshida, T., Matias-Peralta, H., Yusoff, F., Toda, T. \& Othman, B. R. (2012). Zooplankton research in Malaysia: current status and future prospects. Coastal Marine Science 35(1), 208-213.

Yoshida, T., Toda, T., Yusoff, F. \& Ross, O. (2006). Seasonal variation of zooplankton community in the coastal waters of the Straits of Malacca. Coastal Marine Science 30 (1), 32 0-327.

Zakaria, H. Y., Hassan, A. M., Abo-Senna, F. M. \& El-Naggar, H. A. (2016). Abundance, distribution, diversity and zoogeography of epipelagic copepods off the Egyptian Coast (Mediterranean Sea). Egyptian Journal of Aquatic Research 42(4), 459-473 DOI: https://doi.org/10.1016/j.ejar.2016.11.001

Zuraire, M., Cob, Z. C., Toda, T., Othman, B. H. R. \& Yoshida, T. (2018) Seasonal changes in abundance of four acartia species (copepoda, calanoida) in the coastal waters of Peninsular Malaysia; relationship with monsoon transition. Regional Studies in Marine Science 22, 101 111. DOI: https://doi.org/10.1016/j.rsma.2018.06.007 